\title{
PERSONALIZED MARKET BASKET PREDICTION WITH TEMPORAL ANNOTATED RECURRING SEQUENCE
}

\author{
Mr. S. Sambasivam, M C A, M Phil*, Mr. C. Sujendran, M C A ** \\ *(Professor, Department of Computer Applications, \\ Nandha Engineering College (Autonomous), \\ Erode, Tamil Nadu, India \\ Email: sammy2173@gmail.com) \\ ** (Final MCA, Department of Computer Applications, \\ Nandha Engineering College (Autonomous), \\ Erode, Tamil Nadu, India \\ Email: sujendran2296@gmail.com)
}

$* * * * * * * * * * * * * * * * * * * * * * * *$

\begin{abstract}
:
Market Basket Analysis or MBA is an area of modeling strategies based completely upon the concept that if you buy a positive organization of items, you are more (or less) likely to shop for another organization of items. MBA includes self-control and prediction purchaser's conduct based on expenditure sample of preceding clients. MBA is applied not satisfactory in retail however furthermore in an exceptional wide variety of numerous fields. There are research which factor to MBA and make contributions to developing incomes in lodges manage by imparting more appealing additional services for logo spanking new and ordinary customers. MBA based mostly on multidimensional log it model became used to behavior a take a look at Market basket evaluation is to make a preference of purchasing, sailing or possession of shares in an equity market. Data mining strategies ensure excessive precision of prediction of stock charge movement. In this thesis the use of MBA for improving techniques of arranging merchandise on maintain cabinets changed into identified. Analysis of the maximum commonplace customers' transactions changed into performed. In this undertaking, Market basket prediction, i.e., offering the client a buying listing for the subsequent buy in step with her cutting-edge needs, is this type of offerings. Current approaches are not capable of taking photos at the same time the various factors influencing the purchaser's decision process: co-occurrence, sequentuality, periodicity and recurrency of the bought items. To this aim, this mission defines a sample Temporal Annotated Recurring Sequence (TARS) capable of capture concurrently and adaptively these forms of factors. It define the technique to extract TARS and develop a predictor for subsequent basket named TBP (TARS Based Predictor) that, on top of TARS, is in a position to understand the extent of the patron's shares and suggest the set of maximum critical items. By adopting the TBP the grocery store chains may need to crop tailored suggestions for each character patron which in flip ought to correctly accelerate their purchasing sessions.
\end{abstract}

Keywords - Next Basket Prediction, Market Basket Analysis, Interpretable Model. 


\section{INTRODUCTION}

Detecting buy habits and their evolution in time is a critical mission for powerful advertising policies and engagement strategies. In this context, considered one of the most promising centers retail markets can offer to their clients is basket prediction, i.e., the automatic forecasting of the following basket that a consumer will purchase. A powerful basket recommender can act as a shopping list reminder suggesting the objects that the purchaser could probable need.

A hit realization of this application calls for indepth information of an individual's shopping behavior [1]. The purchasing patterns of individuals evolve in time and can experience modifications because of each environmental reasons, like seasonality of merchandise or retail rules, and private reasons, like weight loss program adjustments or shift in personal preferences.

Thus, a exceptional method to basket prediction must be adaptive to the evolution of a patron's behavior, the recurrence of her buy patterns, and their periodic adjustments. This undertaking proposes the Temporal Annotated Recurring Sequences (TARS), adaptive styles which model an individual's purchasing behavior by 4 main traits.

First, TARS keep in mind the co-occurrence: a client systematically purchases a fixed of items together. Secondly, TARS version the sequentiality of purchases, i.e., the fact that a purchaser systematically purchases a fixed of objects after any other one.

Third, TARS remember periodicity: a customer can systematically make a sequential purchase simplest in precise durations of the year, because of environmental factors or personal reasons. Fourth, TARS recall the recurrency of a sequential purchase at some point of each duration, i.e., how frequently that sequential purchase appears all through a patron's length of the year.

Modelling these four aspects - co-occurrence, sequentiality, periodicity and recurrence-is fundamental to stumble on an individual's shopping conduct and its evolution in time. On one hand, future wishes rely on the wishes already satisfied: what a consumer will buy relies upon on what she already purchased.

On the alternative hand, the wishes of a patron depend upon her specific conduct, i.e., habitual purchases she makes over and over. Far from being static, shopping behavior are tormented by both endogenous and personal factors. For this reason, periodicity is an essential characteristic of an adaptive model for basket prediction. This assignment exploits the TARS to assemble a parameter-loose TARS Based Predictor (TBP) which solves the basket prediction problem and presents a basket recommendation as a list of gadgets to be reminded inside the next

This assignment demonstrates the effectiveness of our approach via extracting the TARS for thousands of clients in 3 large-scale real-global datasets. One of the main homes of TARS is their interpretability, which permits retail chains to benefit beneficial insights about the clients' purchasing patterns. It shows that TARS may be used to infer critical characteristics of products, like seasonality and inter-purchase times, which can be without difficulty interpreted via both a simple mathematical notation and a visible

Then, it evaluates TBP with a repertoire of modern techniques and shows that:

(i) TBP outperforms existing strategies.

(ii)TBP can predict up to the subsequent 20 baskets.

(iii) The great of TBP's predictions stabilizes after about 36 weeks. TARS and TBP are usercentric approaches: given a purchaser, they best use the patron's individual information to be expecting her destiny baskets.

This aspect eases the customers' personal data control and permits for growing tailor-made recommenders that can run on private mobile devices.

It is likewise reviewed and classified the related paintings on transactional statistics mining for predictions and guidelines. Next basket prediction is an application of recommender systems based on implicit feedback where simplest effective observations (e.g., purchases or clicks) are available, and no specific preferences (e.g., ratings) are expressed. The implicit comments are given in a 
shape of sequential transactional records acquired through monitoring the users' conduct over time, e.g. A retail store information the transactions of customers through fidelity cards.

Next basket prediction is mainly aimed at the development of effective recommender systems (or recommenders). Recommenders may be categorized into general, sequential, pattern-based totally, and hybrid recommenders. General recommenders are based on collaborative filtering and produce recommendations for a purchaser based on general clients' preferences. They do no longer don't forget any sequential records (i.e., which item is bought after which) and do now not adapt to the customers' latest purchases. In contrast, sequential recommenders are based totally on Markov chains and produce tips for a patron exploiting sequential facts and current purchase]. Pattern primarily based recommenders base predictions on common item sets extracted from the buy history of all clients while discarding sequential records Pattern based approaches often take advantage of or make bigger the Apriori set of rules for extracting the styles.

\section{LITERATURE REVIEW}

In this paper [1] the authors said that in the very last year's many accurate desire guide systems have been constructed as black boxes, this is as structures that cover their internal commonplace sense to the This lack of clarification constitutes both a realistic and an ethical trouble. The literature reports many approaches aimed towards overcoming this vital weakness once in a while at the price of scarifying accuracy for interpretability. The programs in which black container choice systems can be used are various, and each method is commonly advanced to offer a solution for a specific problem and, as a consequence, delineating explicitly or implicitly its very own definition of interpretability.

The goal of this paper is to provide a type of the main problems addressed in the literature with admire to the notion of clarification and the form of black field machine. Given a problem definition, a black container type, and a desired rationalization this survey want to assist the researcher to discover the proposals more useful for his very own The proposed type of procedures to open black container models have to additionally be beneficial for placing the many studies open questions in perspective.

The final decade has witnessed the upward thrust of ubiquitous opaque choice structures. These black field structures take benefit of sophisticated gadget getting to know fashions to expect person statistics that might additionally be sensitive. It can consider credit score, insurance hazard, health status, as examples. Machine getting to know algorithms construct predictive fashions which may be capable of map consumer features proper into a class (very last results or selection) thanks to learning

This gaining knowledge of technique is made viable via the virtual lines that human beings leave at the back of them at the equal time as performing regular activities (e.g., movements, purchases, comments in social networks, etc.). This giant amount of statistics may additionally moreover include human biases and prejudices. Thus, desire fashions found out on them may additionally inherit such biases, probably main to unfair and wrong decisions.

Despite divergent opinions amongst legal scholars regarding the real scope of those clauses [6, $7,10]$, each person consents that the need for the implementation of this type of precept is urgent and that it represents in recent times a large open Without an enabling technology able to explaining the good judgment of black boxes, the right to a proof will continue to be a "lifeless letter". By relying on sophisticated gadget studying models educated on huge datasets way to scalable, highoverall overall performance infrastructures, risk to create and use choice systems that do now no longer certainly This impacts not only statistics on ethics, but additionally on safety and on business liability.

Likewise, the use of device gaining knowledge of models in medical research, for example in medicine, biology, socio-financial sciences, requires a proof not simplest for agree with and reputation of results, but additionally for the sake of the openness of scientific discovery and the improvement of research. As a consequence, clarification is on the coronary heart of a 
responsible, open records science, all through multiple industry sectors and clinical disciplines.

Different clinical agencies studied the trouble of explaining system studying selection models. However, every community addresses the trouble from a particular attitude and gives a one of a kind meaning to clarification. Most of the works inside the literature come from the machine learning and information mining communities.

Existing works have a tendency to provide just a widespread review of the trouble highlighting unanswered questions and issues. On the alternative hand, other works focus on particular components like the impact of representation formats on comprehensibility [10], or the interpretability issues in term of blessings and drawbacks of selected Consequently, after recognizing 4 categories of issues and a hard and fast of approaches to offer an explanation, chosen to organization the methodologies for opening and know-how black field predictors by thinking about concurrently the hassle they're facing, the magnificence of answers proposed for the explanation, the type of statistics analyzed .

\section{Need for Interpretable Models}

Which are the real problems requiring interpretable models and explainable predictions? In this section, they briefly report some instances showing how and why black packing containers may be dangerous. Indeed, delegating decisions to black containers without the opportunity of an interpretation can be critical, can create discrimination and consider issues. Training a classier on ancient datasets, reporting human decisions, could cause the invention of endemic preconceptions [10]. Moreover, considering the fact that these rules can be deeply concealed in the skilled classifier, they hazard considering, maybe unconsciously, such practices and prejudices as preferred rules. They are warned about a growing "black field society" [10], governed by "secret algorithms blanketed by way of business secrecy, criminal protections, obfuscation, so that intentional or unintended discrimination will become invisible and mitigation.
One of the most vital open problems is that, until now, there may be no settlement on what evidence. Indeed, a few works offer as explanation a hard and fast of rules, others a selection tree, others a prototype (especially within the context of images). It is obvious that the research pastime on this discipline completely ignored the significance of reading a popular and not unusual formalism for outlining an evidence, identifying which might be the residences that an explanation need to guarantee, e.g. , soundness, completeness, compactness and comprehensibility.

\section{MODULES}

\section{A. Association Rule Mining}

In this part, Using tweet collection and first 50 larger frequency phrases are in use. Then explicit users inside the twitter also are experimental out.

\section{B. Co-Review Graph Construction}

In this module from precise users in the tweet are discovered out. Same Key word present in two topics of exceptional users is located, and then nodes and one side are formed inside the graph. Thus the full graph is constructed. During side addition, co-occurrence matter is also observed out and set as area weight.

\section{Finding Cliques to Get Fraud Users}

In this component, from the complete diagram constructed, cliques are creating out with minimum 5 nodes in them. These cliques denote the users who are densely connected. These users are treated as fraud users.

\section{Remove Nodes with Edge Weights below Threshold So Normal Users Are Treated As Non-Fraud Users}

In this part, one node, all restrictions are full. If all the edge weights are lower the given threshold values, it means the user is giving rating less times only. The user is treated as normal user. 


\section{EXISTING WORKS}

The market basket Method to integration scheme that allows adapting external heterogeneous data resources for healthy to existed advice device. The integration scheme is primarily based on numerous marketplace basket analysis methods, such as association rules, collaborative filtering and clusterization. For the experimental examine, it located datasets with transaction of information of supermarkets' customers. The existing model defines metrics to evaluate the nice of constructed recommendation gadget and behavior experiments evaluating the authentic and adapted recommendation

Results display the potential to enhance the firstclass of recommendation device by the use of extra heterogeneous facts sources. However, the selected datasets were extremely special and do now not allow us to acquire the anticipated results. The main end result of the examiner is that understood the similarly ways of studies that it's far necessary to locate more appropriate datasets and improve the used strategies.

\section{DRAWBACKS OF EXISTING SYSTEM}

- Only Item -to -item collaborative filtering and recommendations are generated based on a similarity of clients transaction details.

- Improve the quality of constructed RS by using additional data sources

- Common groups of rules by using clusterization methods and filtering of nonconforming parts of data.

- Do not find missing date of customer Transaction data

- Does not identifying missing item set with last transaction data.

- Do not predicated missing item with loss of profit by customer purchase transaction dataset.

\section{PROPOSED SYSTEM}

The Temporal Annotated Recurring Sequences (TARS), adaptive styles which model an individual's purchasing conduct by four number one characteristics. First, TARS recall the cooccurrence: a purchaser systematically purchases a hard and speedy of gadgets together. Secondly, TARS model the sequentiality of purchases, i.e., the truth that a customer systematically purchases a hard and fast of objects after every exclusive one. Third, TARS undergo in thoughts periodicity: a customer can systematically make a sequential buy first-class specifically periods of the year, because of environmental factors or non-public Fourth, TARS remember the recurrency of a sequential buy sooner or later of each period, i.e., how often that sequential buy appears within the route of a consumer's period of the year. Modelling these 4 aspects - co-incidence sequentiality, periodicity and recurrency - is vital to discover an individual's shopping conduct.

\section{ADVANTAGES}

- Any Image type can be taken for text extraction.

- Edge detection plays an important role in it.

- Find missing date of customer Transaction data

\section{CONCLUSION}

Some fraudulent builders deceptively increase the search rank and reputation in their apps (e.g., via fake evaluations and bogus set up counts), even as malicious builders use app markets as a release pad for his or her malware. The motivation for such behaviors is effect: app popularity surges translate into financial advantages and expedited malware proliferation.

This task seeks to identify every malware and seek rank fraud subjects in Google Play. This combination isn't always arbitrary: it posit that malicious developers inn to look rank fraud to enhance the effect of their malware. Unlike present solutions, this venture builds this work at the 
declaration that fraudulent and malicious behaviors leave at the back of telltale signs on app markets.

The task has brought FairPlay, a tool to detect each fraudulent and malware Google Play apps. The experiments at the twitter posts, have proven that a excessive percentage of fraud customers are found. In addition, it showed FairPlay's capacity to find out non-fraud customers also.

\section{REFERENCES}

[1] R. Guidotti, A. Monreale, S. Ruggieri, F. Turini et al., "A survey of methods for explaining black box models," ACM Computing Surveys (CSUR), vol. 51, no. 5, p. 93, 2018.

[2] Y.-A. de Montjoye, E. Shmueli, S. S. Wang, and A. S. Pentland, "openpds: Protecting the privacy of metadata through safe answers," PloS one, vol. 9, no. 7, p. e98790, 2014.

[3] M. Vescovi, C. Perentis, C. Leonardi, B. Lepri, and C. Moiso, "My data store: toward user awareness and control on personal data," in Ubicomp. ACM, 2014, pp. 179-182.

[4] K. Christidis et al., "Exploring customer preferences with probabilistic topic models," in ECML-PKDD, 2010.

[5] Adomavicius, G., Tuzhilin, A.: Toward the next generation of recommender systems: a survey of the state-of the-art and possible extensions, Knowledge and Data Engineering, IEEE Transactions, vol. 17, 734_749 (2005).

[6] B. Goodman and S. Flaxman. Eu regulations on algorithmic decision-making and a right to explanation. In ICML workshop on human interpretability in machine learning (WHI 2016), New York, NY. http://arxiv. org/abs/1606.08813 v1, 2016.

[7] S. Wachter, B. Mittelstadt, and L. Floridi. Why a right to explanation of automated decision-making does not exist in the general data protection regulation. International Data Privacy Law, 7(2):76-99, 2017.

[8] G. Comand`e. Regulating algorithms regulation? first ethico-legal principles, problems, and opportunities of algorithms. In Transparent Data Mining for Big and Small Data, pages 169-206. Springer, 2017.

[9] A. Weller. Challenges for transparency. ArXiv preprint arXiv: 1708.01870, 2017.

[10] J. M. Hofman, A. Sharma, and D. J. Watts. Prediction and explanation in social systems. Science, 355(6324):486488, 2017. 